\section{Panorama de definição e implementação da Política Nacional de Informação e Informática em Saúde}

\author{
An overview of the definition and implementation \\ of the Brazilian National Policy on Health Data and \\ Information Technology
}

\author{
Definición general y aplicación de la Política \\ Nacional de Información e Informática en Salud
}

\begin{abstract}
The This qualitative study aimed to analyze the development and implementation of the Brazilian National Policy on Health Data and Information Technology (NPIIH). We analyzed documents and applied an online questionnaire to the experts involved in developing the policy. The data were submitted to content analysis us ing the categorical thematic modality. The PNIIS is the target of debate and proposals at various levels. Provisions have appeared in parallel to regulate measures on health data and information technology. Community participation in developing this policy and the convergence of laws, standards, resolutions, and policy-making levels in a common and broadly acknowledged and enforced policy are challenges, in addition to linking the public and private sectors. The study concludes that the National Policy on Health Data and Information Technology is making gradual progress, predominantly in theoretical debates, revisions, and updates. There are numerous challenges for its implementation and a prevailing need for legitimation.
\end{abstract}

Health Information Systems; Medical Health Communication; Health Policy
Ricardo Bezerra Cavalcante 1 Marta Macedo Kerr-Pinheiro 2 Eliete Albano de Azevedo Guimarães Richardson Machado Miranda ${ }^{1}$

\section{Resumo}

O Estudo de abordagem qualitativa que teve como objetivo analisar o contexto atual de definição e implementação da Política Nacional de Informação e Informática em Saúde (PNIIS). Realizaram-se pesquisa documental e aplicação de um formulário online aos envolvidos com a definição da PNIIS no cenário nacional. Os dados coletados foram analisados por meio da Análise de Conteúdo, modalidade Temático Categorial. Verificou-se que a PNIIS em construção é alvo de debates e proposições em diversas instâncias. Normativas emergem em paralelo para regulamentar ações de informação e informática em saúde. A participação da população na construção desta política e a convergência das leis, normas, resoluções e instâncias para a formulação de uma política comum, reconhecida, legitimada e implementada são desafios, bem como articulação entre o público e o privado. Conclui-se que o panorama da PNIIS no cenário nacional avança lentamente, predominantemente em debates teóricos, revisões e atualizações. Há muitos desafios para sua implementação e prevalece a necessidade de legitimação.

Sistemas de Informação em Saúde; Informática Médica; Comunicação em Saúde;

Política de Saúde 


\section{Introdução}

A política de informação é definida como um conjunto de práticas que estabilizam e mantêm um regime de informação ${ }^{1}$. Na verdade é uma descrição da origem de um regime de informação, definido como qualquer sistema ou rede mais ou menos estável, no qual a informação flui por meio de determinados canais - de produtores específicos, via estruturas organizacionais específicas, a consumidores ou usuários específicos. São políticas que direcionam as práticas informacionais em contextos diferenciados 2 . Assim, o processo de definição e implementação da Política Nacional de Informação e Informática em Saúde (PNIIS) é o que se pretende compreender neste estudo.

Em relação à PNIIS, mesmo não formalizada, sua evolução no período de 1920 à década de 1990 foi descrita e analisada. A informação em saúde, neste período, foi entendida como estatística médico-sanitária e destinada a alimentar os mecanismos de controle do Estado 3 . Neste contexto vários sistemas de informação isolados foram criados e atribuiu-se grande importância aos avanços tecnológicos em detrimento das definições sobre o conteúdo das informações ${ }^{3}$. Nos anos 1990 a realização das Conferências Nacionais de Saúde e os debates em torno da construção de um novo sistema de saúde brasileiro nortearam as discussões acerca de uma política de informação para a área da saúde $4,5,6$. Neste período, houve a necessidade de cumprir com o que estava previsto na Lei Orgânica da Saúde, principalmente no que tange ao direito à informação, disseminação de informações e a gestão do sistema de informação que se almejava desenvolver 7. Posteriormente, ocorreram outras iniciativas tais como: a Rede Interagencial de Informações para a Saúde (RIPSA), a Rede Nacional de Informações em Saúde (RNIS) e o Cartão Nacional de Saúde (Cartão SUS).

A RIPSA representou uma iniciativa de avaliação da qualidade da informação e de seu uso no processo de gestão, com destaque para a produção de indicadores de saúde. A RNIS destacouse por ser uma ação governamental provendo as secretarias estaduais e municipais com infraestrutura computacional e de conectividade. Em relação ao Cartão SUS tratou-se de licitação internacional para a entrega de produto: sistema cartão. Em paralelo, o DATASUS (Departamento de Informática do SUS) desenvolveu duas soluções de estruturação para suportar o potencial sistema cartão: Cadastro Nacional de Estabelecimentos de Saúde (CNES) e o sistema de cadastramento do indivíduo.
Alguns autores têm demonstrado que a PNIIS é um processo em construção onde se insere a necessidade de apontamentos dos entraves e possibilidades para sua efetivação 3,8 . Persiste o desafio de formulação de uma política de informação em saúde que está atrelada à uma correlação de forças políticas, sociais e econômicas em constante tensão 9. É preciso continuar analisando e registrando o percurso histórico de construção da PNIIS com vistas a sua legitimidade. Compreender como esse processo tem se definido, os desafios que emergem neste contexto e as estratégias de intervenção em desenvolvimento permitirá conhecer os avanços e as limitações da evolução dessa política.

Neste contexto os questionamentos que nortearam esta investigação foram: "Qual o real panorama da PNIIS?" "Quais são os fatores dificultadores de sua implementação?" "Quais estratégias estão sendo propostas visando suplantar estes fatores dificultadores?". Este estudo tem como objetivo analisar o processo de definição da PNIIS, seus avanços e desafios para sua implementação.

\section{Procedimentos metodológicos}

Este estudo de abordagem qualitativa foi desenvolvido em duas etapas: 1a etapa (pesquisa documental) e 2a etapa (aplicação de formulário online).

A justificativa para a escolha da pesquisa documental atrelou-se à necessidade de compreender, a partir do percurso histórico, a evolução da construção da PNIIS e os desafios relacionados. Os documentos podem apresentar riquezas de informações que ampliam o entendimento de objetos cuja compreensão necessita de contextualização histórica e sociocultural ${ }^{10}$. Os documentos que compuseram o corpus da pesquisa foram: relatórios das conferências nacionais de saúde 4,5,6,11,12,13,14; Portarias 15,16; versões da PNIIS 17,18; Relatório do seminário nacional de comunicação, informação e informática em saúde 19; Instrução Normativa 20; Plano Nacional de Saúde 21.

Na segunda etapa utilizou-se de um formulário online para a coleta de dados. O uso de formulários online como técnica de coleta é apropriado para pesquisas qualitativas ou quantitativas, obtendo uma taxa de resposta melhor em relação aos formulários enviados por correio 22,23,24.

Os participantes incluídos nesta etapa foram pesquisadores, gestores e profissionais registrados em instâncias da área de informação e informática em saúde e que tivessem envolvimento com a PNIIS. Assim, foram convidados mem- 
bros das seguintes instâncias: Grupo Técnico de Informação, Saúde e Populações, Associação Brasileira de Pós-graduação em Saúde Coletiva (GTISP/ABRASCO); Sociedade Brasileira de Informática em Saúde (SBIS); Grupo de Trabalho do XI encontro anual da Associação Nacional de Pesquisa e Pós-graduação em Ciência da Informação (ENANCIB) - Informação e Saúde; Comissão de Sistematização da PNIIS (2003 a 2004); Grupo de trabalho de revisão da PNIIS - (2012).

Foram feitas até três tentativas de convite direcionado ao $e$-mail pessoal de cada possível participante. A partir do terceiro convite, caso não respondido, o sujeito era excluído da amostra. Os convites foram enviados a 65 potenciais participantes no período de março de 2013 a janeiro de 2014. Destes, 36 participantes responderam o formulário online sendo 19 (53\%) do sexo masculino e 17 (47\%) do sexo feminino. Quanto ao principal vínculo institucional declarado, $17(47,2 \%)$ na academia, 8 $(22,2 \%)$ em alguma instância do Ministério da Saúde, 5 (13,8\%) em secretarias municipais ou estaduais de saúde, 4 (11,1\%) profissionais vinculados a hospitais e $2(5,5 \%)$ que declaram a sociedade brasileira de informática em saúde como o seu principal vínculo institucional. Ressaltamos que os respondentes também estavam ligados às instâncias GTISP/ABRASCO, SBIS, GT XI-ENANCIB, Comissão de sistematização da PNIIS (2003 a 2004) e Grupo de trabalho de revisão da PNIIS - (2012). Entretanto o mesmo participante estava cadastrado em várias instâncias não sendo possível quantificar o número de sujeitos vinculados a cada instância anterior de forma separada. Isto gerou a necessidade de quantificar os participantes a partir de seu principal vínculo institucional, categorizado em: vinculado à academia, instâncias do ministério da saúde, setores internos às secretarias municipal ou estadual de saúde, vinculados a hospitais ou outro estabelecimento de saúde, outro vínculo institucional.

O instrumento possuía três questões abertas: "A PNIIS no que tange a sua implementação, tem avançado?"; "quais são os fatores dificultadores da implementação da PNIIS?"; "quais as estratégias podem ser propostas visando suplantar estes fatores dificultadores?".

As respostas dos participantes envolvidos neste estudo estão disponíveis no seguinte endereço: http://www.ufsj.edu.br/portal2-repo sitorio/File/pgenf/Tabela\%20com\%20as\%20 respostas $\% 20 \mathrm{dos} \% 20$ participantes $\% 20 \mathrm{da} \% 20$ pesquisa(1).pdf.

Primeiramente, procedeu-se à análise preliminar dos documentos que compuseram o corpus da pesquisa documental. Há cinco di- mensões que compõem a análise preliminar da pesquisa documental 10. São eles: avaliação do contexto em que foi produzido o documento; identificação dos autores, seus interesses e os motivos para a escrita do documento; avaliação da autenticidade e da confiabilidade do texto; avaliação da natureza do texto; identificação dos conceitos-chave e a lógica interna do texto. Todas estas cinco dimensões nortearam a primeira etapa da análise dos documentos selecionados.

Posteriormente, tanto os documentos quanto os dados coletados a partir do formulário online foram analisados a partir da Análise de Conteúdo, que são técnicas de análise de dados linguísticos 25. Propõem-se a identificar os elementos fundamentais da comunicação, sendo enumerados e categorizados. Primeiramente procedeu-se à leitura extenuante de todas as falas dos respondentes. As unidades de registro (recorte das respostas) foram identificadas e interpretadas buscando-se a lógica interna dos fatos e dos relatos, e a articulação das informações com a literatura. Este procedimento gerou os núcleos de sentido caracterizados pela interpretação do pesquisador. A agregação dos núcleos de sentido (junção das falas por semelhanças das respostas) culminou na formação das categorias analíticas. Esta pesquisa foi aprovada por Comitê de Ética em Pesquisa, parecer no 175.119/2012.

\section{Resultados}

A partir da análise de conteúdo foi possível identificar duas categorias: Revelações da pesquisa documental: Avanços, recuos e falta de legitimidade na definição da PNIIS; Percepções sobre o panorama da PNIIS no cenário nacional. Esta última foi decomposta em três subcategorias: (1) avanços relacionados à PNIIS; (2) desafios relacionados à PNIIS que se conformam como obstáculos para sua definição, legitimação e implementação; (3) estratégias propostas com vistas à suplantar os desafios relacionados à PNIIS.

\section{Revelações da pesquisa documental: avanços, recuos e falta de legitimidade na definição da PNIIS}

No início dos anos 2000, na XI Conferência Nacional de Saúde, reconheceu-se que a informação em saúde não tem sido usada adequadamente no SUS apesar de ser fundamental para o aperfeiçoamento das lutas do setor 11. Também se reconheceu a baixa qualidade de dados e a desarticulação dos sistemas de informação nacionais que são departamentalizados e ligados à programas governamentais. Todos estes reco- 
nhecimentos foram fundamentais para constituir o entendimento de que uma política nacional de informação em saúde era necessária. Foi promulgada a seguir a Portaria no 355, de 13 de setembro de 2002, que criou o Comitê Tripartite de Informação e Informática em Saúde (CTIIS) no âmbito da Comissão Intergestores Tripartite (CIT). No Art. 4o do documento foram definidas as funções do CTIIS, sendo uma delas a formulação de uma PNIIS 15.

Do texto inicial da PNIIS iniciado em 2003 até o texto final apresentado na 12a Conferência Nacional de Saúde alguns trâmites ocorreram. Em julho de 2003 a primeira versão da PNIIS foi apresentada durante o VII Congresso da Associação Brasileira de Pós-graduação em Saúde Coletiva, onde alguns dirigentes do Ministério da Saúde apresentaram os fundamentos da PNIIS. Este foi um momento de debates que proporcionou o aperfeiçoamento do texto da proposta. A partir dos debates originou-se a versão $1.1 \mathrm{da}$ PNIIS que foi apresentada à Câmara Técnica da Comissão Intergestores Tripartite, contando com as contribuições e o envolvimento do Conselho Nacional de Secretários de Estado de Saúde e o Conselho Nacional de Secretários Municipais de Saúde. A partir das discussões da versão 1.1 da PNIIS nos municípios e estados, juntamente com outras instâncias, produziu-se a versão 1.2 sendo debatida no II Seminário Nacional sobre Informação em Saúde da Fundação Oswaldo Cruz (Fiocruz), realizado em outubro de 2003. Nesse mesmo período o documento foi disponibilizado para consulta pública.

Após a consulta pública e os reordenamentos do documento, produziu-se a versão 1.3. Essa versão foi então apresentada na 12ạ Conferência Nacional de Saúde 8. A necessidade de democratizar o acesso à informação, comunicação e informática em saúde foi a grande proposta que emergiu a partir desta conferência com vistas a ampliar o controle social.

Ao fim da 12a Conferência originou-se a versão 2.0 da PNIIS. Em síntese, o Ministério da Saúde em parceria com diversas instâncias internas e externas fomentou a criação do documento PNIIS 12, relevante para a definição da política de informação em construção.

Outro evento importante foi o Seminário Nacional de Comunicação, Informação e informática em Saúde, promovido pelo Conselho Nacional de Saúde, ocorrido em Brasília em 2005. Ressaltase que, anteriormente, ocorreram também encontros regionais ao longo do ano de 2005 nas diversas regiões brasileiras. As reflexões e proposições desenvolvidas nestes espaços foram socializadas no seminário ocorrido em Brasília. O produto esperado no evento foi o de legitimar e publicizar a Política Nacional de Comunicação, Informação e Informática em Saúde 19.

Contrariando os esforços e debates empreendidos, a PNIIS de 2004 não chegou a ser regulamentada, e por sua vez, não alterou o panorama nacional relacionado às ações de informação e informática em saúde 18. Apenas no ano de 2008, aparece de forma sucinta, numa proposição na 13ạ Conferência Nacional de Saúde: "que o Ministério da Saúde, por meio do DATASUS, promova a retomada da discussão da PNIIS” 13 (p. 35).

Apesar da PNIIS ainda não estar formalizada e legitimada, a incorporação de tecnologias da informação na área da saúde continuava a se desenvolver de forma descontrolada. Uma forma de controlar, quando necessário, e avaliar os processos de infraestrutura, foi editada a Instrução Normativa/SLTI/MP no 4, de 12 de Novembro de 2010. Nesta Instrução há o reconhecimento da incorporação das Tecnologias da informação no setor público, porém de forma disciplinada 20 .

Em 31 de agosto de 2011 publicou-se a Portaria no 2072 que redefiniu o Comitê de Informação e Informática em Saúde (CIINFO) atribuindo-lhe competências, que juntas, pudessem fortalecer a PNIIS 16 .

Na 14ạ Conferência Nacional de Saúde a necessidade de definição de uma Política de informação em saúde continuou sendo proposta na $12^{\mathrm{a}}$ diretriz. Esta política deveria assegurar a “gestão participativa e eficaz ao SUS" 14 (p. 73). Para assegurar a definição da política de informação foram propostas 15 ações relacionadas a: aprimoramento e integração dos sistemas de informação do SUS; informatização dos serviços de saúde; implantação definitiva do Cartão SUS; criação de um prontuário nacional para os usuários do SUS; implementação da telessaúde e telemedicina, bem como ampliação do uso da Educação à Distância (EaD).

O Plano Nacional de Saúde (PNS) 2012-2015 é outro documento que emerge neste percurso histórico. No que diz respeito à PNIIS, a mesma é citada no PNS apenas na diretriz 12, entretanto ao se observar as metas relacionadas a esta mesma diretriz, não há referências explícitas à PNIIS 21.

Ainda em 2012 uma nova versão do documento da PNIIS em construção foi disponibilizado para consulta pública com vistas à participação da sociedade em sua elaboração. No período sugestões e críticas foram realizadas no sentido de aprimorar a PNIIS ${ }^{18}$. As diretrizes da PNIIS de 2012 propostas neste novo documento englobam: (a) estrutura e fortalecimento da área de informação e informática em saúde no país; (b) desenvolvimento de tecnologias; (c) disseminação da informação; (d) formação e capa- 
citação de recursos humanos em informação e informática em saúde e o desenvolvimento de redes de centro colaboradores/pesquisas na área de informação e informática em saúde; (e) fortalecimento da articulação interfederativa no que tange a PNIIS; (f) desenvolvimento de um arcabouço ético-legal.

Por fim, é importante ressaltar que a PNIIS proposta atualmente apresenta uma tendência de alinhamento com o movimento de $e$-health (e-saúde) em desenvolvimento no cenário internacional. Inclusive é proposto no documento que a PNIIS passe a chamar política nacional de $e$-saúde 18 .

\section{Percepções sobre o panorama da PNIIS no cenário nacional}

Os discursos dos participantes foram agrupados em três subcategorias que revelaram suas percepções sobre o panorama da PNIIS no contexto brasileiro.

A primeira subcategoria "avanços relacionados à PNIIS” destaca a evolução quanto à “adoção de padrões de interoperabilidade como a troca de informação de saúde suplementar" (P24), ao "investimento em infraestrutura tecnológica” (P25) e à “institucionalização de comitês específicos para debater a temática” (P25). Os respondentes também apontaram certo avanço na "compreensão do que se configura, efetivamente como uma Política Nacional de Informação e Informática em Saúde" (P4), considerando importante "alinhamento entre as ações do Ministério e os princípios da PNIIS" (P9), bem como a "revisão da PNIIS incorporando aspectos fundamentais nas novas diretrizes" (P17).

A segunda subcategoria revela os "desafios relacionados à PNIIS que se conformam como obstáculos para sua definição, legitimação e implementação". Esta categoria destacou-se com maior frequência entre as demais demonstrando que os sujeitos entendem o panorama da PNIIS como desafiador. Os respondentes destacaram que o "aparelho administrativo brasileiro é paquidérmico" (P19), "precisa ser urgentemente reformado" (P19) e "os trâmites burocráticos são tão longos e morosos que as soluções ficam obsoletas" (P30). Também destacaram que há " $a$ falta de prioridade política para a incorporação de tecnologia da informação e comunicação, planejamento de médio e longo prazos" (P6); "não há investimentos em infraestrutura para acesso à Internet" (P6); “atraso tecnológico e o próprio estágio de desenvolvimento do SUS, com sua desigualdade entre regiões brasileiras" (P7). Outras dificuldades que foram apontadas estiveram relacionadas com o fato de que a PNIIS necessita de maior "envolvimento de todas as esferas de governo e da sociedade civil" (P4); "a sua aprovação final e edição oficial pelo Ministério da Saúde em conjunto com os Conselhos" (P7), bem como não seja uma ação isolada e descontinuada de diversas instâncias no cenário brasileiro. Também foram destacados como desafios o fato de haver "pouquíssimos espaços de educação formal na área de informática em saúde no Brasil" (P14); a exigência de "produção de dados, mas não incorpora a discussão e reflexão sobre os mesmos" (P32); a "maior ênfase em soluções tecnológicas do que em organização, métodos e pessoas" (P7) $\mathrm{e}$ ainda as relações de interesse representadas pelo avanço do complexo econômico de tecnologia da informação/telecomunicação "sobre o SUS como um grande mercado promissor, favorecido pelo esvaziamento das instâncias públicas de ITIS" (P10), bem como pelo "envolvimento intenso dos pesquisadores com a indústria" (P33).

Além dos desafios relacionados à PNIIS identificados pelos participantes há também a proposição de estratégias visando suplantar estes desafios. Isto fez emergir uma terceira categoria analítica, Estratégias propostas com vistas à suplantar os desafios relacionados à PNIIS. Os respondentes destacaram a necessidade de "investimento em infraestrutura, parque de equipamentos de informática" (P27); "investimento em recursos humanos" (P26); "educação formal em nível de graduação e pós-graduação na área de Informática em Saúde" (P14), bem como a necessidade de "fomentar o desenvolvimento da gestão da informação e do conhecimento" (P21). Foi proposto "diminuir a burocracia e dar continuidade aos programas já iniciados" (P13); "maior inserção e participação dos profissionais de informação junto às equipes multiprofissionais" (P25); e ainda a proposição de uma "articulação coordenada com setores da ciência e tecnologia, indústria e comércio, educação e participação social” (P7). Os participantes destacaram que "o consenso no entendimento da PNIIS é fundamental” (P8), porém “ a voz não pode ser apenas a dos especialistas, é preciso somar outros olhares que procedem de outras áreas" (P19). Por fim, foi proposto o alinhamento da PNIIS com outros documentos, tais como: "O documento a visão de e-Saúde para o Brasil recentemente entregue ao Ministério da Saúde" (P7); as "Dimensões estratégicas para o desenvolvimento da Informação e Tecnologia de Informação em Saúde contempladas no 20 Plano Diretor de Informação e Tecnologia de Informação em Saúde" (P10); e a necessidade de a PNIIS ser "mais aderente à Política Brasileira Nacional de Metrologia e Normalização” (P31). 


\section{Discussão dos resultados}

Os resultados revelaram que a PNIIS, além de avançar lentamente em sua definição, enfrenta diversos obstáculos para sua implementação, dentre eles um modelo político de informação obsoleto e inapropriado para a sociedade da informação e do conhecimento. No Brasil, o modelo de gerenciar a informação foi, durante muitos anos, centrado no autoritarismo e seu ensejo pelo controle, e as questões envolvendo a necessidade do sigilo sempre foram utilizadas no Brasil como justificativa para tratar a informação como posse irrestrita de governo 3,26. Atualmente a PNIIS sustenta um modelo político de informação em saúde, onde em parte ainda há o controle do governo sobre o fluxo informacional, a construção de sistemas de informação departamentalizados, a produção de dados armazenados em centrais de informação e a incipiente democratização do acesso à informação.

No percurso histórico da PNIIS, nos documentos analisados, há o reconhecimento do direito à informação e a necessidade de que esta política seja uma construção coletiva, conhecida por todos e pactuada nas diversas esferas do SUS. Por um lado isto pode ser considerado um avanço, visto o modelo político brasileiro que sempre considerou a informação como posse irrestrita de governo 3,27 . Porém é necessário promover condições para que a população usuária se aproprie das suas informações, reconheça o seu direito e estabeleça a sua cidadania. O desafio é o de institucionalizar o uso e a disseminação da informação produzida na intenção de apoiar as decisões tomadas. Além disso, deve-se considerar outros desafios como o de ampliar e popularizar os meios de distribuição da informação, de expandir a rede de disseminação, e de desenvolver políticas e ações que promovam o controle social ${ }^{28}$. O arcabouço teórico, legal, ético e as condições necessárias para controle social são igualmente importantes para que o direito à informação em saúde se estabeleça. Ressalta-se que a informação em saúde deve ser entendida como um bem público caracterizado por uma produção social, um direito de todos e um dever do estado brasileiro. Além disso, deve estar sob gestão colegiada, democrática e disponível ao exercício do controle social 29,30. Isto difere do modelo político de informação como posse irrestrita de governo que utiliza o sigilo como justificativa de necessidade e a incipiente preocupação em garantir o acesso a informação para a população e profissionais.

Quanto à pactuação da PNIIS, foi apontado que os municípios brasileiros ainda sofrem com infraestrutura precária, poucos recursos huma- nos, atraso tecnológico, baixa conectividade, baixa qualificação profissional, desigualdade e desorganização do SUS nas diversas regiões brasileiras, bem como um financiamento insuficiente. Pactuar o que não pode ser cumprido devido aos motivos descritos anteriormente é expor uma política ao seu insucesso. Outros autores também afirmam que as instâncias públicas relacionadas ao campo da informação e informática em saúde, em sua maioria, ainda carecem de estruturação 29,30.

Diversos debates e proposições relacionados à PNIIS foram destacados, porém sem continuidade e com pouca legitimação. O debate em diversas instâncias assim como a publicação de portarias e resoluções relacionadas à PNIIS pode ser analisado como um processo construtivo e um avanço necessário, pois a construção de políticas de informação atuais são processos complexos que envolvem múltiplos atores e níveis de interação 31. Contudo, ainda é desafiador que na definição da PNIIS ocorra a convergência das leis, normas, resoluções e instâncias para a formulação de uma política comum, legitimada e implementada. É preciso considerar que o debate em torno da PNIIS transcenda a responsabilização de instâncias e a promulgação de portarias ou resoluções isoladas. Políticas de informação necessitam ser documentadas, para se registrar o que deve ser implementado, por que e por quem, criando sua tangibilidade e tornando o uso da informação pertinente e eficaz 29 .

Verificou-se a proposição do Plano Diretor de Informação e Tecnologia de Informação em Saúde: 2008-2012 (PlaDITIS), o alinhamento da PNIIS com outras políticas (metrologia e normalização) e até mesmo o entendimento de que a PNIIS esteja associada a uma estratégia maior, a $e$-health. Os PlaDITIS, documentos construídos pelo GTISP/ABRASCO, proporcionaram a análise do contexto nacional acerca do campo da informação e informática em saúde, principalmente reconhecendo as dificuldades existentes, bem como propondo estratégias de solução. Considera-se o esforço do GTISP na criação dos PlaDITIS com o objetivo de intervir sobre o campo da informação e informática em saúde, porém alguns autores defendem que os planos são temporais, não são legislados e não implicam a participação dos poderes executivos e legislativo 32,33. A política de informação, pelo contrário, exige um posicionamento do governo na definição de normas, leis e reconhecimento em todo o território. Além de possuir uma temporalidade de longo prazo, há um imediatismo nos planos que dificulta o reconhecimento das políticas como processos de mediação e de tradução 32,33 . 
Acreditamos que os PlaDITIS, como uma formulação não governamental, mas coletiva e plural, não substituem a PNIIS em construção, pelo contrário, a reforçam e exigem a sua legitimação no cenário nacional. Endossando esta afirmação o próprio GTISP aponta o PlaDITIS como o resultado de um esforço que buscou construir uma agenda que consubstancie a formulação, implementação e avaliação da PNIIS, enquanto requisito estratégico para o aperfeiçoamento do SUS 30 .

Sobre o alinhamento da PNIIS com outras políticas, preocupa-nos o fato de supostamente serem políticas paralelas e, em alguns aspectos, tratarem as mesmas diretrizes da PNIIS com encaminhamentos diferenciados. Os constantes discursos verificados "política de normalização e metrologia"; "política de informática"; "política de software público"; "política de $e$-health" evocam esta preocupação.

O termo $e$-health ou e-saúde emergiu dos discursos como uma estratégia maior, em detrimento da PNIIS, devendo esta última estar associada a esta estratégia. A Organização Mundial da Saúde (OMS) definiu, por meio da Resolução WHA58.28, o conceito de $e$-health como o uso das tecnologias de informação e comunicação para a saúde ${ }^{34}$. Alguns exemplos são citados, tais como as tecnologias que proporcionam o suporte à assistência a pacientes, pesquisa, educação e capacitação da força de trabalho em saúde, bem como a monitoração e avaliação em saúde. Em relação a $e$-health, mesmo sendo um movimento reconhecido internacionalmente, ainda sim se deve entender melhor a sua apropriação, limites, possibilidades para o país e para o SUS. É preciso compreender o real discurso impregnado em seus documentos, suas possibilidades e seus limites para o cenário nacional. Outra investigação necessária entra no campo epistemológico, pois seria possível esta mudança de termos PNIIS para Política de $e$-saúde? Tratam do mesmo objeto? São conceitos semelhantes? Abrangem, de fato, outros aspectos além da tecnologia? $\mathrm{Ou}$ ultrapassam este limite histórico? A política proposta estaria, de fato, legislando sobre um regime informacional e incorporando os elementos de uma política de informação? Vale ressaltar que o termo política de informação, dentro do campo da Ciência da Informação, possui delimitação conceitual 1,2,35,36.

Outro desafio é a necessidade de considerar as relações de poder existentes durante a construção da PNIIS. As mesmas foram verificadas no discurso sobre o avanço dos conglomerados de Tecnologia da informação e Telecomunicações sobre o espaço público e nos discursos relacionados às intencionalidades de atores em diversos espaços. As relações de poder, impregnadas nos discursos e ações relacionadas à PNIIS, certamente têm envolvido a sua definição e influenciado os rumos de sua legitimação 29,30. É preciso considerar que o poder é exercido nas relações sociais, e isto pode ser feito a partir da informação e seus processos. A própria constituição da PNIIS é envolvida por discursos que são manifestações destas relações de poder. As informações ali impregnadas refletem direcionamentos e intencionalidades.

O campo da informação e informática em saúde no Brasil tem sido um cenário para atuação das empresas privadas de tecnologia 29. Neste contexto, o espaço público torna-se um excelente mercado consumidor de soluções tecnológicas. É de se esperar que o debate na formulação de uma política de informação também sofra a atuação de intencionalidades que tendem a manter espaços de atuação do mercado privado. Isto precisa ser considerado. O espaço público deve ser capaz de confrontar estes interesses particulares e transformá-los em benefícios para a população, gestores e profissionais. O desmantelamento sofrido pelo espaço público, no que tange às questões de informação e informática em saúde, precisam ser superados 3,29 .

O Estado brasileiro é capaz de se organizar com vistas a assumir a hegemonia do desenvolvimento tecnológico ao invés de sucumbir aos interesses privados. Além da reorganização e estruturação dos setores de informação e tecnologia da informação no país, emerge, neste contexto, o papel da academia e outras instituições científicas públicas. São espaços de desenvolvimento tecnológico e inovação que podem, se potencializados, configurar-se como apoiadores do cenário público. O intuito é estruturar o Estado para atender aos interesses do SUS, seus gestores e a população assistida, mas também é possível compartilhar com o mercado as possibilidades de desenvolvimento tecnológico. Estudo 34 já aponta para a estruturação do cenário público no que tange aos setores de informação e informática em saúde. Porém são necessários estudos aprofundados deste processo de estruturação, e aqui evocamos a necessidade de entender a política de informação que tem norteado estes espaços, o regime de informação, os critérios de incorporação tecnológica, dentre outros. Insistimos em propor a necessidade de ir além do imperativo tecnológico na definição da PNIIS. É preciso que haja certo equilíbrio entre artefatos técnicos e conteúdos emancipadores.

$\mathrm{O}$ que se vislumbra e que constitui um fator comum e limitador do avanço das políticas de informação é o enfoque dado às questões instrumentais, tecnicistas e tecnológicas como priori- 
dade, ainda hegemônicas 1. Esta visão simplista de que a tecnologia é um fator de grandes mudanças pode empobrecer o discurso da própria política e renegar os processos atrelados, que são fundamentais para sua implementação. Para romper com esta ótica é preciso incorporar as várias dimensões do entorno da convergência tecnológica digital: o conteúdo e sua mediação, os processos atrelados, as pessoas envolvidas e a estrutura.

\section{Considerações finais}

A análise dos discursos relacionados à PNIIS revela um panorama com avanços lentos e repleto de desafios para sua definição. Os avanços são predominantemente teóricos, mas há algumas diretrizes desta política em implementação no cenário nacional. Prevalece a necessidade de legitimação da PNIIS. Apesar da cristalização aparente da PNIIS é possível verificar avanços nos debates e no amadurecimento acerca da concepção sobre a informação em saúde.

O avanço do debate deve ser transformado em ações de articulação dos diferentes sistemas de informação em um verdadeiro sistema de saú- de que facilite o acesso, a interlocução e a adaptação às novas necessidades sociais. E, acima de tudo a retomada do processo sobre a definição da PNIIS exige sua explicitação pública, em lei, como base para o alcance dos objetivos propostos pelo Ministério da Saúde.

Finalizando este trabalho emergem algumas problemáticas. É preciso analisar a proposta de $e$-health brasileira, seus desafios, possibilidades, aproximações e divergências em relação à PNIIS e sua capacidade de legislar sobre o regime informacional estabelecido. É necessário investigar como os principais atores/decisores/criadores de políticas nas esferas do SUS compreendem uma política de informação, e se suas compreensões não estariam esvaziando a sua importância e real necessidade.

Em relação às limitações do estudo buscamos as percepções dos sujeitos sobre o objeto PNIIS. Isto foi sustentado pela metodologia qualitativa utilizada. Evidentemente, os resultados não devem ser universalizados, pois representam as percepções dos participantes incluídos nesta pesquisa. É a maneira como estes respondentes observam o objeto PNIIS e não um olhar de todos os profissionais da área de Informação e Informática em Saúde. 


\section{Resumen}

Este estudio cualitativo tuvo como objetivo analizar el contexto actual de la definición e implementación de la Política Nacional de Información e Informática en Salud (PNIIS). Hemos llevado a cabo la investigación documental y la aplicación de un formulario en línea para los que participan en la definición de PNIIS en la escena nacional. Los datos obtenidos fueron analizados mediante análisis de contenido, modalidad temática categórica. La PNIIS en construcción es objeto de debates y propuestas en varios casos. Las normas emergen en paralelo para regular las acciones de información y de la informática de la salud. La participación popular en la construcción de esta política y la convergencia de las leyes, reglamentos, resoluciones y órganos para la formulación de una política común, reconocida así como la interacción entre lo público y lo privado. Se concluye que las perspectivas para PNIIS en la escena nacional avanza lentamente, sobre todo en los debates teóricos, revisiones y actualizaciones. Hay muchos retos para su implementación, pero sigue siendo una necesidad legítima.

Sistemas de Información en Salud; Informática

Médica; Comuncación en Salud; Politica de Salud

\section{Colaboradores}

R. B. Cavalcante e E. A. A. Guimarães participaram de todas as etapas da pesquisa e elaboração do artigo. M. M. Kerr-Pinheiro e R. M. Miranda colaboraram na elaboração do projeto, coleta e análise dos dados e redação final do artigo.

\section{Agradecimentos}

O presente estudo foi financiado pelo Conselho Nacional de Desenvolvimento Científico e Tecnológico (CNPq).

\section{Referências}

1. Frohmann B. Taking information policy beyond information science: applying the actor network theory. In: 23th Annual Conference, Canadian Association form Information; 1995. http://www.ual berta.ca/dept/slis/cais/frohmann.htm (acessado em 24/Abr/2014).

2. Rowlands I, Eisenschitz T, Bawden D. Frame analysis as a tool for understanding information policy. Journal of Information Science 2002; 28:31-8.

3. Branco MAF. informação em saúde: uma ciência e suas políticas em uma nova era. Rio de Janeiro: Editora Fiocruz; 2006

4. Ministério da Saúde. Conferência Nacional de Saúde. In: Anais da 8a Conferência Nacional de Saúde, 1987. http://bvsms.saude.gov.br/bvs/ publicacoes/8conf_nac_anais.pdf (acessado em 24/Abr/2012).
5. Ministério da Saúde. Conferência Nacional de Saúde. Relatório Final da 9a Conferência Nacional de Saúde, 1992. http://bvsms.saude.gov.br/bvs/ publicacoes/9_conferencia_nacional_saude_relato rio_final.pdf (acessado em 24/Abr/2012).

6. Ministério da Saúde. Conferência Nacional de Saúde. Relatório final da $10^{a}$ Conferência Nacional de Saúde, 1998. http://conselho.saude.gov.br/bi blioteca/Relatorios/relatorio_10.pdf (acessado em 24/Abr/2012).

7. Brasil. Lei no 8.080 de 19 de setembro de 1990. Dispõe sobre as condições para a promoção, proteção e recuperação da saúde, a organização e o funcionamento dos serviços correspondentes e dá outras providências. Diário Oficial da União 1990; 20 set. 
8. Cavalcante RB, Kerr MMP. Política Nacional de Informação e Informática em Saúde: avanços e limites atuais. Perspectivas em Gestão \& Conhecimento $2011 ; 1: 106-19$.

9. Moraes IHS, Vasconcellos MM. Política Nacional de Informação, Informática e Comunicação em Saúde: um pacto a ser construído. Saúde Debate 2005; 29:86-98.

10. Cellard AA. Análise documental. In: Poupart J, Deslauriers J-P, Groulx L-H, Laperrière A, Mayer R, Pires AP, organizadores. A pesquisa qualitativa: enfoques epistemológicos e metodológicos. Petrópolis: Editora Vozes; 2008. p. 295-316.

11. Ministério da Saúde. Conferência Nacional de Saúde. Relatório final da 11ạ Conferência Nacional de Saúde, 2002. http://conselho.saude.gov.br/bi blioteca/Relatorios/relatorio_11.pdf (acessado em 15/Out/2012).

12. Ministério da Saúde. Conferência Nacional de Saúde. Relatório final da 12a Conferência Nacional de Saúde, 2004. http://bvsms.saude.gov.br/bvs/ publicacoes/rel_final_12CNS.pdf (acessado em 15/ Out/2012).

13. Ministério da Saúde. Conferência Nacional de Saúde. Relatório final da 13a Conferência Nacional de Saúde, 2008. http://conselho.saude.gov.br/ biblioteca/Relatorios/13cns_M.pdf (acessado em 23/Abr/2012).

14. Ministério da Saúde. Conferência Nacional de Saúde. Relatório final da 14a Conferência Nacional de Saúde, 2012. http://conselho.saude.gov. br/14cns/docs/Relatorio_final.pdf (acessado em 23/Abr/2012).

15. Fundação Nacional de Saúde/Ministério da Saúde. Portaria no 355, de 13 de setembro de 2002. Cria, no âmbito da Comissão Intergestores Tripartite (CIT), o Comitê de Informação e Informática em Saúde. Diário Oficial da União 2002; 16 set.

16. Ministério da Saúde. Portaria no 2.072, de 31 de agosto de 2011. Redefine o Comitê de Informação e Informática em Saúde (CIINFO/MS) no âmbito do Ministério da Saúde. Diário Oficial da República Federativa do Brasil 2011; 2 set.

17. Ministério da Saúde. Política Nacional de Informação e Informática em Saúde. Versão 2.0. Inclui deliberações da 12a Conferência Nacional de Saúde, 2004. http://bvsms.saude.gov.br/bvs/publicacoes/ PoliticaInformacaoSaude29_03_2004.pdf (acessado em 15/Out/2012).

18. Ministério da Saúde. Comitê de Informação e Informática em Saúde, Ministério da Saúde. Política Nacional de Informação e Informática em Saúde, 2012. http://www.isc.ufba.br/arquivos/2012/Poli tica_Nacional_de_Informacao_e_Informatica_em Saude.pdf (acessado em 23/Abr/2012).

19. Conselho Nacional de Saúde, Ministério da Saúde. Relatório do seminário nacional de comunicação, informação e informática em saúde para o exercício do controle social, 2006. http://conselho.saude. gov.br/biblioteca/livros/relatorioseminario_miolo. pdf (acessado em 15/Out/2012).
20. Secretaria de Logística e Tecnologia da Informação, Ministério do Planejamento, Orçamento e Gestão. Instrução Normativa/SLTI/MP no 4 de 12 de novembro de 2010. Dispõe sobre o processo de contratação de Soluções de Tecnologia da Informação pelos órgãos integrantes do Sistema de Administração dos Recursos de Informação e Informática (SISP) do Poder Executivo Federal. http://www.governoeletronico.gov.br/biblioteca/ arquivos/instrucao-normativa-no-04-de-12-denovembro-de-2010/download (acessado em 24/Abr/2012).

21. Subsecretaria de Planejamento e Orçamento, Secretaria-Executiva, Ministério da Saúde. Plano Nacional de Saúde - PNS: 2012-2015. http://bvsms. saude.gov.br/bvs/publicacoes/plano_nacional_ saude_2012_2015.pdf (acessado em 23/Abr/2012).

22. Kehoe CM, Pitkow JE. Surveying the territory: GVU's five WWW user surveys. The World Wide Web Journal 1996; 1:77-84. http://www.cc.gatech. edu/gvu/user_surveys/papers/w3j.html (acessado em 22/Nov/2000).

23. McCollough D. Web-based market research, the dawning of a new era. Direct Marketing 1998; 61:36-9.

24. Foina A. Métodos de aquisição de dados quantitativos na internet: o uso da rede como fonte de dados empíricos. Ciência \& Trópico 2002; 30:283-96.

25. Bardin L. Análise de conteúdo. 4a Ed. Lisboa: Edições 70; 2010.

26. Gruman M. Lei de acesso à informação: notas e um breve exemplo. Revista Debates 2012; 6:97108.

27. Batista CL. Informação pública: controle, segredo e direito de acesso. Intexto 2012; 26:204-22.

28. Lima CRA, Leal CD, Dias EP, Gonzales FL, Santos HL, Silva MEM, et al. A experiência de disseminação de informações em saúde. In: Ministério da Saúde, organizador. A experiência brasileira em sistemas de informação em saúde. v. 1. Brasília: Ministério da Saúde; 2009. p. 109-28. (Série B. Textos Básicos de Saúde).

29. Moraes IHS. Política, tecnologia e informação em saúde: a utopia da emancipação. Salvador: Casa da Qualidade Editora; 2002.

30. Grupo Técnico de Informação em Saúde e População, Associação Brasileira de Pós-Graduação em Saúde Coletiva. Plano Diretor de Informação e tecnologia de informação em saúde: 2008-2012. http://www.abrasco.org.br/grupos/ arquivos/20100210132738.pdf (acessado em 15/ Mar/2009).

31. Higino AFF, Araújo RF, Scott CSP. Construção de políticas de informação: aspectos epistemológicos e metodológicos. Liinc em Revista Online 2008; 4:286-302. http://revista.ibict.br/liinc/index.php/ liinc/article/view/283.

32. Marques RM, Pinheiro MMK. Política de informação nacional e assimetria de informação no setor de telecomunicações brasileiro. Perspectivas em Ciência da Informação 2011; 16:65-91. 
33. Pinheiro MMK. Processo de transformação das políticas de informação no estado informacional. Tendências da Pesquisa Brasileira em Ciência da Informação 2010; 3:113-26.

34. World Health Organization. Building foundations for eHealth: progress of Member States: report of the WHO Global Observatory for eHealth. Geneva: World Health Organization; 2006.

35. Gomez MNG. Novos cenários políticos para a informação. Ciência da Informação 2002; 31:27-40.
36. Braman S. Change of state; information, policy and power. Cambridge: The MIT Press; 2006.

37. Santos AF, Ferreira JM, Queiroz NR, Magalhães Júnior HM. Estruturação da área de informação em saúde a partir da gerência de recursos informacionais: análise de experiência. Rev Panam Salud Pública 2011; 29:409-15.

Recebido em 18/Jun/2014

Versão final reapresentada em 05/Dez/2014

Aprovado em 08/Dez/2014 